\title{
Model Development and Inverse Compensator Design for High Speed Nanopositioning
}

\author{
Ralph C. Smith*, Murti V. Salapaka ${ }^{\dagger}$, Andrew Hatch*, Joshua Smith ${ }^{\ddagger}$ and Tathagata De $^{\dagger}$ \\ ${ }^{*}$ Center for Research in Scientific Computation, North Carolina State University, Raleigh, NC 27695 \\ ${ }^{\dagger}$ Electrical Engineering Department, Iowa State University, Ames, IA 50011 \\ ‡Department of Physics, North Carolina State University, Raleigh, NC 27695
}

\begin{abstract}
This paper focuses on the development of constitutive models, commensurate system models, and inverse compensator construction for high speed nanopositioning in atomic force microscopes (AFM). All current AFM employ either stacked or cylindrical piezoceramic actuators for both longitudinal and transverse positioning of the sample. An inherent property of these materials is the presence of hysteresis and constitutive nonlinearities, even at the low drive levels employed for angstromlevel resolution. At low frequencies, standard feedback mechanisms effectively attenuate the hysteresis, whereas noise at high frequencies diminishes the efficacy of feedback and leads to unacceptable accuracy. In this paper, we discuss modeling techniques which provide a first step toward high speed nanopositioning for applications ranging from macroscopic product evaluation to realtime imaging of biological processes.
\end{abstract}

\section{Introduction}

Piezoceramic transducers are employed in a large range of applications requiring angstrom-level positioning due to their high set point accuracy, large dynamic range, and relatively small temperature sensitivity. They are presently employed in all atomic force microscope (AFM) designs for both lateral and vertical positioning of samples and are being considered as drive elements for future nanopositioning and nanoconstruction applications. The positive attributes of piezoceramic (PZT) materials are complemented, however, by hysteresis and constitutive nonlinearities inherent to the compounds even at low drive levels and frequencies. During low frequency operation, conventional feedback laws successfully mitigate these effects, thus leading to the phenomenal success of the AFM technology. However, at the higher frequencies required for applications, including real-time imaging of biological processes and efficient product evaluation, two phenomena degrade the accuracy achieved by present control techniques: (i) the piezoceramic materials exhibit increased hysteresis, and (ii) noise to data ratios increase to levels where feedback laws are augmenting noise rather than attenuating hysteresis and nonlinear dynamics. This necessitates the development of control designs which utilize physical models and model inverses to quantify and compensate for hysteresis rather than relying solely on feedback laws based on inherently inaccurate state estimates.

The models designed for this role must satisfy competing objectives; they must be sufficiently accurate to predict the nonlinear material dynamics under varying operating conditions and they must be sufficiently efficient to allow real-time implementation. In this paper, we discuss the development of a methodology for constructing hysteresis models and model inverses for piezoceramics materials based on free energy properties of the materials. This provides an inverse compensator framework suitable for subsequent control design. The motivating application is taken to be AFM design which permits substantially increased scan rates. However, the models and model inverses apply to general piezoceramic compounds and are appropriate for general applications involving high accuracy, high speed, nanopositioning.

To illustrate issues involved with model, compensator, and control development, consider the AFM design depicted in Figure 1. The sample is positioned using either a stacked actuator utilizing $d_{33}$ motion or a cylindrical actuator employing $d_{31}$ motion. As the sample is moved

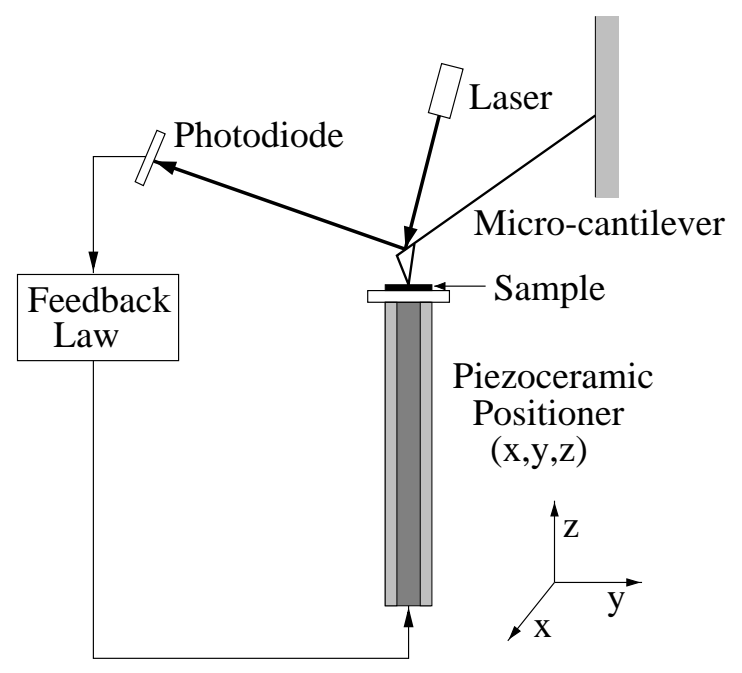

Figure 1. Configuration of a prototypical AFM. 
laterally along a prescribed $x-y$ grid, displacements in response to changing forces between the cantilever tip and sample are measured using the photodiode. A feedback law is then used to raise or lower the sample, again via the piezoceramic transducer, to maintain constant forces. Details regarding the construction and applications utilizing atomic force microscopes and scanning tunneling microscopes (STM) can be found in [4].

The accuracy of the device is intimately related to the accuracy of the piezoceramic positioning elements. While piezoceramic compounds exhibit high set point accuracy, they also exhibit hysteresis in the relation between input fields or voltages and the strains or displacements generated by the device as shown in Figure 2. As illustrated in [8], the degree of hysteresis increases with increasing frequency which, in combination with increased noise to data ratios, exacerbates the difficulties associated with control design.

In recent papers, a variety of techniques have been employed to construct models and inverse compensators as a prelude to control design for high speed nanopositioning. Domain wall theory was employed in [9] while models based on Preisach techniques were considered in [10] for quantifying hysteresis inherent to the positioning mechanism in an AFM. While both techniques yield accurate models for quantifying and compensating for hysteresis, both suffer deficiencies which limit their utility for broadband control design. The domain wall models are highly accurate for symmetric hysteresis loops but require substantial modification for biased regimes; hence they are difficult to implement when transient dynamics are significant. The Preisach models accommodate biased hysteresis loops but do so at the cost of a large number of nonphysical parameters. This limits their utility for high speed implementation. Further-

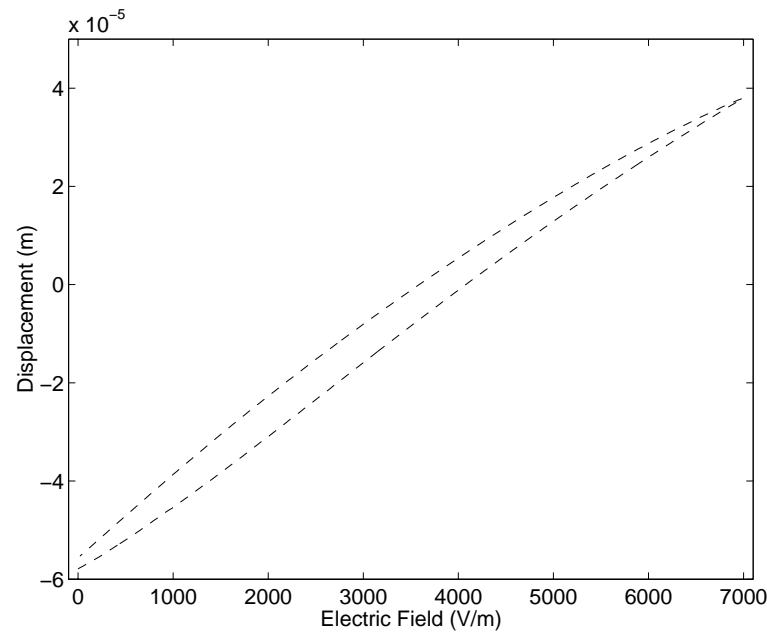

Figure 2. Relation between the input field $E$ and displacements generated by the PZT positioning mechanism in an AFM. more, the Preisach theory requires substantial extension to accommodate variable frequencies, temperatures and stress effects.

The model and inverse compensator developed in this paper are based on energy properties of the materials. In Section 2, free energy theory, in conjunction with stochastic homogenization techniques, is used to develop constitutive equations for the constituent piezoceramic materials. In Section 3, these constitutive relations are incorporated in classical rod models to provide a dynamic model for the stacked actuator. The performance of the model is illustrated in Section 4 through comparison with the experimental data plotted in Figure 2. The development of an inverse compensator appropriate for inclusion in a feedforward control loop is summarized in Section 5.

\section{Constitutive Relations}

When modeling the constitutive behavior of the piezoceramic stacked actuator, we assume that the stressstrain relation is adequately modeled by linear expressions whereas the map between input fields $E$ or voltages $V$ and the generated polarization $P$ is nonlinear and hysteretic. Moreover, we assume that the material is biased through poling to operate in regimes where the relation between the polarization and strains $\varepsilon$ is approximately linear. This latter assumption is motivated by a number of classical references $[2,5]$ and experiments are being designed to establish the validity of this assumption for the regimes under consideration.

To model the nonlinear map between $E$ and $P$, and to provide a framework which yields appropriate constitutive relations, we first establish Helmholtz and Gibbs energy relations at the domain level. This yields a mesoscopic model appropriate for homogeneous, single crystal compounds with uniform effective fields. The effects of material nonhomogeneities, polycrystallinity, and variable effective fields are then incorporated through stochastic distributions to obtain macroscopic models suitable for system characterization and inverse compensator construction for subsequent control design. The discussion of the $E-P$ hysteresis model follows the development in $[11,12,13]$ and details regarding its construction can be found in those sources.

For fixed temperature conditions with no applied stresses $\sigma$, the Helmholtz energy exhibits piecewise quadratic behavior and hence an appropriate model is

$$
\psi(P)= \begin{cases}\frac{1}{2} \eta\left(P+P_{R}\right)^{2} & , P \leq-P_{I} \\ \frac{1}{2} \eta\left(P-P_{R}\right)^{2} & , P \geq P_{I} \\ \frac{1}{2} \eta\left(P_{I}-P_{R}\right)\left(\frac{P^{2}}{P_{I}}-P_{R}\right) & ,|P|<P_{I} .\end{cases}
$$



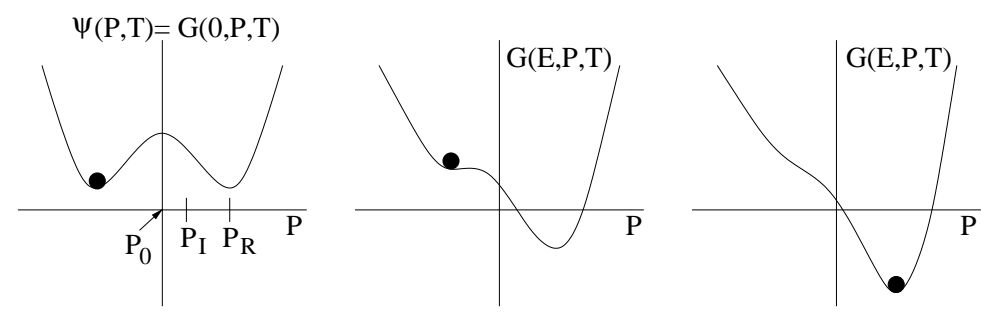

(a)
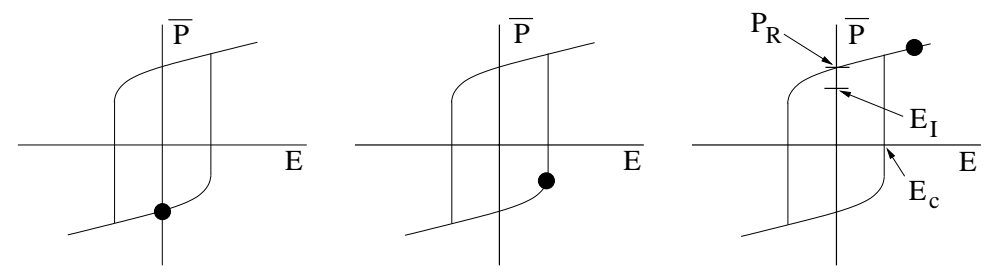

(b)

Figure 3. (a) Helmholtz energy $\psi$ and Gibbs energy $G$ for increasing field $E$. (b) Local polarization $\bar{P}$ as a function of $E$ for a homogeneous, isotropic material.

As illustrated in Figure $3, P_{I}$ and $P_{R}$ respectively denote the inflection point and polarization at which the positive local minimum of $\psi$ occurs. The point $P_{R}$ is also the local remanence polarization at the domain level. Finally, the fact that $\eta$ is the reciprocal of the slope in the $E-P$ relation after switching can be utilized to determine initial parameter values when establishing the model for a given piezoceramic compound and application.

In the absence of applied stresses $\sigma$, it is illustrated in [12] that an appropriate choice of the Gibbs energy is

$$
G(E, P)=\psi-E P
$$

where the latter term incorporates the electrostatic energy due to an applied field $E$. The behavior of $G$ given by (2) is illustrated for positive fields in Figure 3.

To incorporate ferroelastic coupling, we employ the extended Helmholtz relation

$$
\psi_{e}(P, \varepsilon)=\psi(P)+\frac{1}{2} Y^{P} \varepsilon^{2}-Y^{M} \gamma \varepsilon P
$$

and corresponding Gibbs energy

$$
G(E, P, \varepsilon)=\psi(P)+\frac{1}{2} Y^{P} \varepsilon^{2}-Y^{M} \gamma \varepsilon P-E P-\sigma \varepsilon
$$

where $\psi$ is specified by (1). Here $Y^{P}$ denotes the Young's modulus at constant polarization and $\gamma$ is a ferroelastic coupling coefficient. We note that the final term in (4) incorporates the elastic energy.

In the formulation (1) for the Helmholtz energy, it is implicitly assumed that dipoles assume two orientations which we denote as positive and negative. The expected polarization due to positively oriented dipoles is

$$
\left\langle P_{+}\right\rangle=\int_{P_{0}}^{\infty} P \mu(G) d P
$$

where

$$
\mu(G)=C e^{-G V / k T}
$$

quantifies the probability of achieving the energy level $G$. Here $V$ denotes a representative volume, $k$ is Boltzmann's constant, and $C$ is an integration constant chosen to guarantee a probability of 1 when integrated over all feasible dipole orientations. Evaluation of $C$ yields

$$
\begin{aligned}
&\left\langle P_{+}\right\rangle= \frac{\int_{P_{0}}^{\infty} P e^{-G(E, P, T) V / k T} d P}{\int_{P_{0}}^{\infty} e^{-G(E, P, T) V / k T} d P} \\
&\left\langle P_{-}\right\rangle=\frac{\int_{-\infty}^{P_{0}} P e^{-G(E, P) V / k T} d P}{\int_{-\infty}^{P_{0}} e^{-G(E, P) V / k T} d P} .
\end{aligned}
$$

The fraction of dipoles having positive and negative orientations are respectively quantified by the evolution equations

$$
\begin{aligned}
& \dot{x}_{+}=-p_{+-} x_{+}+p_{-+} x_{-} \\
& \dot{x}_{-}=-p_{-+} x_{-}+p_{+-} x_{+} .
\end{aligned}
$$

The likelihoods of switching from positive to negative, and conversely, are specified by

$$
\begin{aligned}
& p_{+-}=\sqrt{\frac{k T}{2 \pi m V^{2 / 3}}} \frac{e^{-G\left(E, P_{0}, T\right) V / k T}}{\int_{P_{0}}^{\infty} e^{-G(E, P, T) V / k T} d P} \\
& p_{-+}=\sqrt{\frac{k T}{2 \pi m V^{2 / 3}}} \frac{e^{-G\left(E, P_{0}, T\right) V / k T}}{\int_{-\infty}^{P_{0}} e^{-G(E, P, T) V / k T} d P}
\end{aligned}
$$


where $m$ is the mass of the lattice volume $V$. For homogeneous materials with constant effective field $E_{e}=E$, the local average polarization is then given by

$$
\bar{P}=x_{+}\left\langle P_{+}\right\rangle+x_{-}\left\langle P_{-}\right\rangle .
$$

For regimes in which operating time scales make thermal activation negligible, asymptotic relations can be employed to simplify the polarization kernel (9) since jumps can be assumed to occur instantaneously (see $[12,13])$ for details). In this regime, $\bar{P}=P_{\min }$ where $P_{\text {min }}$ is the solution to

$$
\frac{\partial G}{\partial P}=0 .
$$

This yields the piecewise linear definition

$$
\left[\bar{P}\left(E, \varepsilon ; E_{c}, \xi\right)\right](t)=\left\{\begin{array}{l}
{\left[\bar{P}\left(E, \varepsilon ; E_{c}, \xi\right)\right](0)} \\
\frac{E}{\eta-2 Y^{P} \gamma \varepsilon}-\frac{P_{R} \eta}{\eta-2 Y^{P} \gamma \varepsilon} \\
\frac{E}{\eta-2 Y^{P} \gamma \varepsilon}+\frac{P_{R} \eta}{\eta-2 Y^{P} \gamma \varepsilon}
\end{array}\right.
$$

for the respective cases $\{\tau(t)=\emptyset\},\{\tau(t) \neq \emptyset$ and $\left.E(\max \tau(t))=-E_{c}\right\},\{\tau(t) \neq \emptyset$ and $E(\max \tau(t))=$ $\left.E_{c}\right\}$. The local coercive field is given by

$$
E_{c}=\eta\left(P_{R}-P_{I}\right) .
$$

The transition points $\tau$ are specified by

$$
\tau(t)=\left\{t \in\left(0, T_{f}\right] \mid E(t)=-E_{c} \text { or } E(t)=E_{c}\right\},
$$

and

$$
\left[\bar{P}\left(E, \varepsilon ; E_{c}, \xi\right)\right](0)=\left\{\begin{array}{l}
\frac{E}{\eta-2 Y^{P} \gamma \varepsilon}-\frac{P_{R} \eta}{\eta-2 Y^{P} \gamma \varepsilon} \\
\xi \\
\frac{E}{\eta-2 Y^{P} \gamma \varepsilon}+\frac{P_{R} \eta}{\eta-2 Y^{P} \gamma \varepsilon}
\end{array}\right.
$$

denotes the initial dipole orientation for respective initial fields $\left\{E(0) \leq-E_{c}\right\},\left\{-E_{c}<E(0)<E_{c}\right\}$ or $\left\{E(0) \geq E_{c}\right\}$.

The relations (9) or (10) quantify the hysteretic relation between $E$ and $P$ for homogeneous, single crystal compounds having uniform effective field $E_{e}=E$. To extend this mesoscopic model to macroscopic regimes involving nonhomogeneous, polycrystalline compounds with variable effective fields, we consider certain parameters to be manifestations of underlying distributions rather than constant values. To incorporate variations in the lattice structure, we assume that the local coercive field $E_{c}$ specified by (11) is log-normally distributed; that is, we assume that it has the density

$$
f\left(E_{c}\right)=c_{1} e^{\left.-\ln \left(E_{c} / \bar{E}_{c}\right) / 2 b\right]^{2}}
$$

where, if $b$ is small compared with $\bar{E}_{c}, \bar{E}_{c}$ denotes a mean coercive field at which dipoles switch. Effective field effects (see $[1,6]$ ) are incorporated by assuming that the actual field at the domain level has the density

$$
\widehat{f}(\mathcal{E})=c_{2} e^{-(\mathcal{E}-E)^{2} / \bar{b}} .
$$

The macroscopic polarization model is then

$$
P(E)=C \int_{0}^{\infty} \int_{-\infty}^{\infty} \bar{P}\left(E, \varepsilon ; E_{c}, \xi\right) f\left(E_{c}\right) \widehat{f}(\mathcal{E}) d \mathcal{E} d E_{c}
$$

with $\bar{P}$ given by (9) or (10). It is detailed in [11] that the formulation (14) provides an energy basis for certain Preisach representations with the difference that temperature and frequency dependencies are incorporated in the kernel of (14) rather than the parameters as is the case for Preisach models.

The elastic constitutive relation is determined from the equilibrium condition

$$
\frac{\partial G}{\partial \varepsilon}=0
$$

which yields

$$
\sigma=Y^{M} \varepsilon-Y^{M} \gamma P .
$$

In combination, the nonlinear polarization relation (14) and linear elastic relation (15) quantify the constitutive behavior of the piezoceramic materials for operating regimes pertinent to nanopositioning.

\section{Stacked Actuator Model}

It was noted in previous discussion that nanopositioners are typically comprised of stacked actuators utilizing $d_{33}$ motion or cylindrical shells employing $d_{31}$ motion. We outline here the construction of a model for the stacked actuator and refer the reader to [9] for theory illustrating the development of an analogous model for cylindrical transducers.

As will be detailed in Section 4, the cross-sectional area of stacked actuators is small compared with the length which permits consideration of rod models that quantify longitudinal displacements. One end of the transducer is fixed while the other is either free or constrained by an elastic load. For this development, we consider a free end condition but note that the model can be easily modified to accommodate a mass or elastic loading at the free end (e.g., see [3]). The rod is assumed to have cross-sectional area $A$ and length $\ell$, and displacements in the longitudinal ( $x$-axis) are denoted by $u$. The density, Young's modulus and Kelvin-Voigt damping parameters are respectively denoted by $\rho, Y^{P}$ and $c_{D}$.

Force balancing and enforcement of boundary conditions yields the strong form of the model

$$
\begin{aligned}
& \rho A \frac{\partial^{2} u}{\partial t^{2}}=\frac{\partial \mathcal{N}}{\partial x} \\
& u(t, 0)=\mathcal{N}(t, \ell)=0
\end{aligned}
$$

where $\mathcal{N}=\int_{A} \sigma d A$ denotes the force resultant. To quantify $\sigma$ in a manner which incorporates Kelvin-Voigt 
damping, we employ an extension of (15) in which it is assumed that stresses are proportional to a linear combination of strain, strain rate and polarization. With the linear strain relation $e=\frac{\partial u}{\partial x}$, this yields

$$
\mathcal{N}=Y^{P} A \frac{\partial u}{\partial x}+c_{D} A \frac{\partial^{2} u}{\partial x \partial t}-Y^{P} A \gamma P(E)
$$

where the polarization is specified by (14).

To define a weak or variational form of the model which is appropriate for well-posedness analysis, approximation, or control design, the state $u$ is considered in the state space $X=L^{2}(0, \ell)$ with the inner product

$$
\langle\phi, \psi\rangle_{X}=\int_{0}^{\ell} \rho A \phi \psi d x .
$$

The space of test functions is taken to be $V=H_{\ell}^{1}(0, \ell)=$ $\left\{\psi \in H^{1}(0, \ell) \mid \psi(0)=0\right\}$ with the inner product

$$
\langle\phi, \psi\rangle_{V}=\int_{0}^{\ell} Y^{P} A \phi^{\prime} \psi^{\prime} d x .
$$

Multiplication by test functions followed by integration then yields the weak form

$$
\begin{aligned}
& \int_{0}^{\ell} \rho A \frac{\partial^{2} u}{\partial t^{2}} \psi d x+\int_{0}^{\ell} Y^{P} A \frac{\partial u}{\partial x} \frac{\partial \psi}{\partial x} d x \\
& +\int_{0}^{\ell} c_{D} A \frac{\partial^{2} u}{\partial x \partial t} \frac{\partial \psi}{\partial x} d x=\int_{0}^{\ell} Y^{P} A \gamma P(E) \frac{\partial \psi}{\partial x} d x
\end{aligned}
$$

which must be satisfied for all $\psi \in V$. Details regarding the approximation of (17) using finite elements to obtain a finite-dimensional system can be found in $[3,9]$.

\section{Model Validation}

To illustrate attributes of the model, we consider the characterization of the stacked actuator used to generate the data plotted in Figure 2. The actuator has a width of $5 \mathrm{~mm}$ and length of $20 \mathrm{~mm}$, and is configured for $d_{33}$, or longitudinal, motion. The Young's modulus and density specified by the manufacturer are $Y^{P}=6.5 \times 10^{10} \mathrm{~N} / \mathrm{m}^{2}$ and $\rho=7730 \mathrm{~kg} / \mathrm{m}^{3}$. The Kelvin-Voigt damping parameter $c_{D}=6.5 \times 10^{7} \mathrm{Ns} / \mathrm{m}^{2}$ and polarization parameters $b=100, \bar{b}=1.178 \times 10^{7}, C=1 \times 10^{4}, \eta=1.14 \times 10^{6}$ and $\bar{E}_{c}=6.374 \times 10^{-9}$ were estimated through a least squares fit to the data.

Displacement data collected at $0.1 \mathrm{~Hz}$ with a $30 \mathrm{~V}$ oscillation about a bias level of $70 \mathrm{~V}$ is plotted in Figure 4 along with the model prediction. It is observed that the model accurately quantifies both the hysteresis and constitutive nonlinearities inherent to the material. Moreover, by employing the algorithms detailed in [13], implementation of the model is sufficiently efficient to permit the development of model-based compensators capable of being implemented in real time.

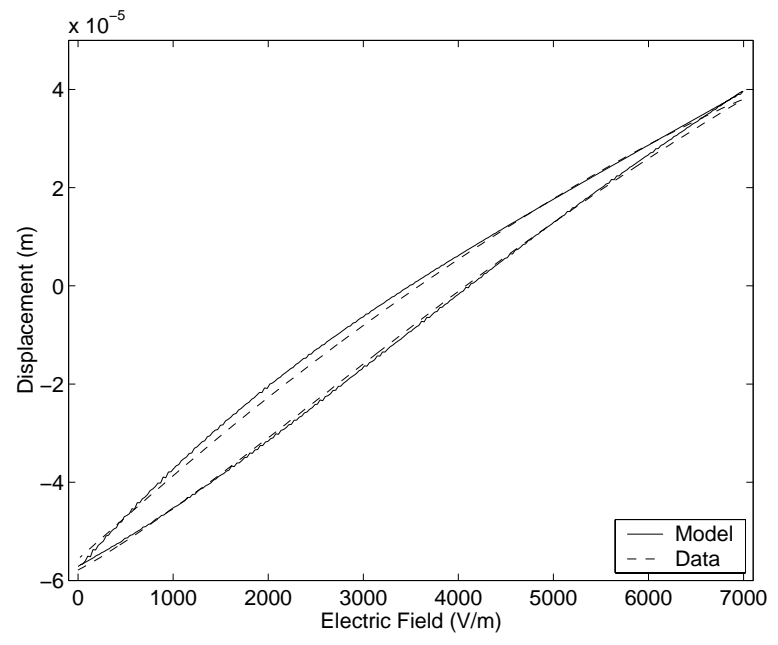

Figure 4. Experimental data collected from the stacked actuator and model prediction.

\section{Model Inverse}

The monotone relation between $E$ and $P$ can be utilized to construct inverse models which specify the field necessary to achieve a given polarization level. These inverse maps can then be employed in feedforward loops to compensate for hysteresis and constitutive nonlinearities in a manner which facilitates linear control design.

The construction of a model quantifying the inverse map between $P$ and $E$ is outlined in Algorithm 1. For given values of $P_{\text {prev }}$ and $P_{\text {new }}$, the relation (14) is used to increment the field until the predicted polarization $P_{t m p}$ has advanced beyond $P_{\text {new }}$, and the final field value $E_{\text {new }}$ is determined through linear interpolation between the final two predicted field values. When implementing the algorithm, the stepsize $\Delta E$ is adaptively updated to ensure that the efficiency of the inverse algorithm is close to that of the forward algorithm.

The inverse model provided by Algorithm 1 is compared with experimental PZT5A data, reported in [12], in Figure 5. The model parameters are the same as those specified for the forward model in [12]. Figure 5 illustrates the accuracy of Algorithm 1 for quantifying the $P-E$ behavior of piezoceramic materials.

\section{Algorithm 1.}

$$
\begin{aligned}
& \text { Specify } E_{\text {prev }}, P_{\text {prev }}, P_{\text {new }} \\
& \text { Specify } \Delta E \\
& d P=P_{\text {new }}-P_{\text {prev }} \\
& E_{\text {tmp }}=E_{\text {prev }}, P_{t m p}=P_{\text {prev }} \\
& \text { while } \operatorname{sgn}(d P)\left(P_{\text {new }}-P_{t m p}\right) \geq 0 \\
& \quad E_{t m p}=E_{t m p}+\Delta E \\
& \quad P_{t m p} \text { given by }(14) \\
& \text { end } \\
& E_{\text {new }} \text { given by linear interpolation }
\end{aligned}
$$




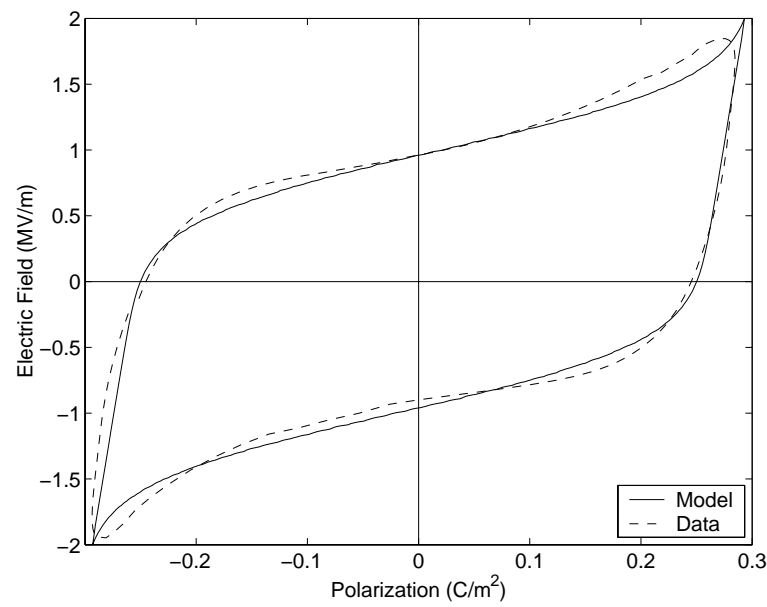

Figure 5. Experimental PZT5A data from [12] and inverse model predicted by Algorithm 1 .

\section{Concluding Remarks}

The model presented here quantifies the hysteresis and constitutive nonlinearities inherent to the piezoceramic positioning elements employed for nanopositioning by characterizing energy properties of the materials. By employing statistical homogenization techniques to construct effective parameters for the models, significant flexibility is imbued in the resulting constitutive relations including minor loop closure under biased operating conditions. Furthermore, this yields models and commensurate inverse relations which are highly efficient and hence promote real-time implementation.

From a control perspective, these models are important since they provide a means of quantifying nonlinear material dynamics in regimes where feedback mechanisms are ineffective due to measurement errors or inaccurate state estimates. The inverse maps can be employed to construct inverse compensators for linear control design as detailed for optimal control laws in [7] and adaptive control techniques in [14]. Current investigations are focused on the development of robust control techniques which employ the inverse compensators outlined in this paper for high accuracy, high speed, nanopositioning.

\section{Acknowledgements}

The research of RCS was supported in part by the Air Force Office of Scientific Research under the grant AFOSR-F49620-01-1-0107 while the support of JS was provided by the NSF grant CMS-0099764.

\section{References}

[1] J.C. Anderson, Dielectrics, Reinhold Publishing Corporation, New York, 1964.
[2] W.G. Cady, Piezoelectricity, McGraw-Hill, New York, 1946.

[3] M.J. Dapino, R.C. Smith and A.B. Flatau, "A structural-magnetic strain model for magnetostrictive transducers," IEEE Transactions on Magnetics, 36(3), pp. 545-556, 2000.

[4] P.K. Hansma, V.B. Elings, O. Marti and C.E. Bracker, "Scanning tunneling microscopy and atomic force microscopy: Application to biology and technology," Science, 242, pp. 209-242, 1988.

[5] T. Ikeda, Fundamentals of Piezoelectricity, Oxford University Press, Oxford, 1990.

[6] T. Mitsui, I. Tatsuzaki and E. Nakamura, An Introduction to the Physics of Ferroelectrics, Gordon and Breach Science Publishers, New York, 1976.

[7] R.C. Smith, "Inverse compensation for hysteresis in magnetostrictive transducers," Mathematical and Computer Modelling, 33, pp. 285-298, 2001.

[8] R.C. Smith, Z. Ounaies and R. Wieman, "A model for rate-dependent hysteresis in piezoceramic materials operating at low frequencies," Proceedings of the SPIE, Smart Structures and Materials 2000, Newport Beach, CA, Volume 3992, pp. 128-136, 2000 .

[9] R.C. Smith and M. Salapaka, "Model development for the positioning mechanisms in an atomic force microscope," International Series of Numerical Mathematics, to appear.

[10] R.C. Smith, M.V. Salapaka and L. Cherveny, "A Preisach model for quantifying hysteresis in an atomic force microscope," Proceedings of the SPIE, Smart Structures and Materials 2002, Volume 4693, pp. 498-504, 2002.

[11] R.C. Smith and S. Seelecke, "An energy formulation for Preisach models," Proceedings of the SPIE, Smart Structures and Materials 2002, Volume 4693, pp. 173-182, 2002.

[12] R.C. Smith, S. Seelecke and Z. Ounaies, "A free energy model for piezoceramic materials," Proceedings of the SPIE, Smart Structures and Materials 2002, Volume 4693, pp. 183-190, 2002.

[13] R.C. Smith, S. Seelecke and Z. Ounaies, "A free energy model for hysteresis in ferroelectric materials," preprint.

[14] G. Tao and P.V. Kokotović, Adaptive Control of Systems with Actuator and Sensor Nonlinearities, John Wiley and Sons, New York, 1996. 